\title{
Sporotrichosis with Bone Involvement: An Alert to an Occupational Disease
}

\author{
Felipe de Carvalho Aguinaga ${ }^{a} \quad$ Beatriz Moritz Trope $^{a}$ \\ Nurimar Conceição Fernandes ${ }^{a} \quad$ Delia Celser Engel $^{b}$ \\ Marcia Ramos-e-Silva ${ }^{a}$ \\ ${ }^{a}$ Sector of Dermatology and Post-Graduation Course and ${ }^{b}$ Sector of Infectious-Parasitary \\ Diseases, University Hospital and School of Medicine, Federal University of Rio de Janeiro, \\ Rio de Janeiro, Brazil
}

\section{Key Words}

Sporotrichosis · Subacute mycosis · Chronic mycosis · Bone

\begin{abstract}
Sporotrichosis is a subacute or chronic mycosis caused by a fungus of the genus Sporothrix, which is found in soil. It can be acquired by trauma to the skin. Bone and joint lesions are very rare. The city of Rio de Janeiro is undergoing an epidemic transmitted by cats, and this should be an alert for the risk to professionals in contact with these animals. The patient was a veterinarian who developed occupational sporotrichosis with osteoarticular involvement transmitted by a cat during a consultation.

(c) 2014 S. Karger AG, Basel
\end{abstract}

\section{Introduction}

Sporotrichosis is a subacute or chronic mycosis caused by fungi of the genus Sporothrix, whose natural reservoir is the soil. It is transmitted through inoculation of the traumatic agent in the skin [1,2]. Osteoarticular lesions are rare [3]. The peculiar epidemiological situation of the city of Rio de Janeiro, where an epidemic of cases transmitted by cats is observed [4], must serve as a warning for the risk of professionals in contact with these animals to acquire the disease. We report a case of occupational sporotrichosis in a veterinarian who had bone involvement. 
Aguinaga et al.: Sporotrichosis with Bone Involvement: An Alert to an Occupational Disease

\section{Case Report}

The patient was a 48-year-old Caucasian female veterinarian who lived in Rio de Janeiro. Whilst working on the medication of a cat with sporotrichosis, her left hand was scratched. It evolved after 4 weeks with the formation of an erythematous papule on the left thumb. After using various antibiotics without improvement, she was referred to our hospital approximately 2 months after onset. Comorbidities included hypertension, insulin-dependent diabetes mellitus, hypothyroidism and heavy smoking.

Upon examination, she presented edema and significant erythema in the distal phalanx of the left thumb, with a distal crusted ulcer area causing detachment of the nail plate (fig. 1, fig. 2). She complained of severe pain requiring opioids for analgesia. Her ipsilateral forearm showed small nodules, slightly erythematous, painful on palpation, following the lymphatic path, more palpable than visible.

Skin biopsy was performed and the material was sent for cultures. Bacterioscopy was negative and the culture for common germs showed growth of Escherichia coli, considered as a contaminant. Bacilloscopy and culture for mycobacteria were negative, whilst fungal culture showed growth of Sporothrix schenckii.

Due to the location of the lesion, the degree of involvement of the phalanx and intense pain, imaging tests were requested to investigate osteoarticular involvement. Plain radiography revealed involvement of the distal phalanx, with the appearance of osteomyelitis (fig. 3), subsequently confirmed by computed tomography. There were no signs of joint involvement. Bone biopsy was performed on the involved phalanx, with the bone fragments sent for culture, and there was recurrent growth of $S$. schenckii.

The conclusion, therefore, was sporotrichosis with bone involvement, and itraconazole $400 \mathrm{mg} /$ day was started for 12 months. The patient progressed well, with complete healing of the lesion and pain relief, remaining in ward attendance.

\section{Discussion}

Sporotrichosis, whose agent is a fungus of the genus Sporothrix, is transmitted especially through traumatic inoculation of its agent into the skin. Classically, the transmission occurs after minor trauma with twigs, branches, thorns and other vegetals. The incubation period varies from 3 days to 3 months [1-3].

Since 1998, the city of Rio de Janeiro has been undergoing a peculiar situation, an epidemic of zoonotic sporotrichosis due to infected cats, which is considered rare in other regions of Brazil and the world $[4,5]$. The increased incidence of sporotrichosis in Rio de Janeiro has been observed for over a decade. From 1998 to 2004, 759 humans and 1,503 cats were diagnosed with sporotrichosis at IPEC-FIOCRUZ, a reference institution of Rio de Janeiro [6]. In the previous period, from 1986 to 1998, only 13 cases were diagnosed in the same center [6]. An increase in the number of cases with extracutaneous involvement was also observed [6]. The situation worsened between 2005 and 2008, when 804 new cases were reported, already being considered the greatest epidemic by zoonotic transmission [7].

Osteoarticular lesions are rare and particularly affect immunocompromised, alcoholic and diabetic patients [8]. The infection can occur by contiguity or hematogenous diffusion, which may manifest from small granulomas to large lytic lesions, similar to osteomyelitis [9]. Only a small number of patients present general symptoms and leukocytosis, whilst the vast majority present increased hemosedimentation rate [8]. 
Sporotrichosis was always considered as an occupational disease [1-3]. In 1926, Foerster [10] drew attention to the fact that the majority of sporotrichosis cases were acquired at work, and that many of them could generate sequels that would compromise labor activities and therefore must have their occupational nature emphasized [3]. Sporotrichosis has been described to be a 'gardener's disease' and to be primarily related to rose planters, but a range of professionals, especially in rural areas, is subject to acquiring the infection, such as farmers, miners, lumberjacks and others $[1,2]$.

The new epidemiology profile of the disease in the city of Rio de Janeiro, however, should be a warning to the risk of contamination of other professionals that could acquire the disease, especially veterinarians. It is believed that it is not necessary for a cat to scratch or bite in order to transmit the disease; thus, professionals should be informed about the risks and the use of personal protective equipment, such as gloves, to manipulate cats that are sick or under suspicion of sporotrichosis.

\section{References}

1 Ramos-e-Silva M, Vasconcelos C, Carneiro S, Cestari T: Sporotrichosis. Clin Dermatol 2007;25:181-187.

2 Barros MB, de Almeida Paes R, Schubach AO: Sporothrix schenckii and sporotrichosis. Clin Microbiol Rev 2011;24:633-654.

3 Carrada-Bravo T: New observations on the epidemiology of sporotrichosis and Sporothrix schenckii complex. Rev Latinoamer Patol Clin 2012;59:88-100.

4 Cordeiro FN, Bruno CB, Paula CD, Motta Jde O: Familial occurrence of zoonotic sporotrichosis. An Bras Dermatol 2011;86(4 suppl 1):S121-S124.

5 Freitas DF, do Valle AC, de Almeida Paes R, Bastos FI, Galhardo MC: Zoonotic sporotrichosis in Rio de Janeiro, Brazil: a protracted epidemic yet to be curbed. Clin Infect Dis 2010;50:453.

6 Schubach A, Barros MB, Wanke B: Epidemic sporotrichosis. Curr Opin Infect Dis 2008;21:129-133.

7 Silva MB, Costa MM, Torres CC, Galhardo MC, Valle AC, Magalhães Mde A, Sabroza PC, Oliveira RM: Esporotricose urbana: epidemia negligenciada no Rio de Janeiro, Brasil [Urban sporotrichosis: a neglected epidemic in Rio de Janeiro, Brazil]. Cad Saude Publica 2012;28:1867-1880.

8 Appenzeller S, Amaral TN, Amstalden EM, Bertolo MB, Neto JF, Samara AM, Fernandes SR: Sporothrix schenckii infection presented as monoarthritis: report of two cases and review of the literature. Clin Rheumatol 2006;25:926-928.

-9 Costa RO, de Mesquita KC, Damasco PS, Bernardes-Engemann AR, Dias CM, Silva IC, Lopes-Bezerra LM: Infectious arthritis as the single manifestation of sporotrichosis: serology from serum and synovial fluid samples as an aid to diagnosis. Rev Iberoam Micol 2008;25:54-56.

10 Foerster HR: Sporotrichosis, an occupational dermatosis. JAMA 1926;87:1605-1609. 


\section{Case Reports in Dermatology}

Aguinaga et al.: Sporotrichosis with Bone Involvement: An Alert to an Occupational Disease

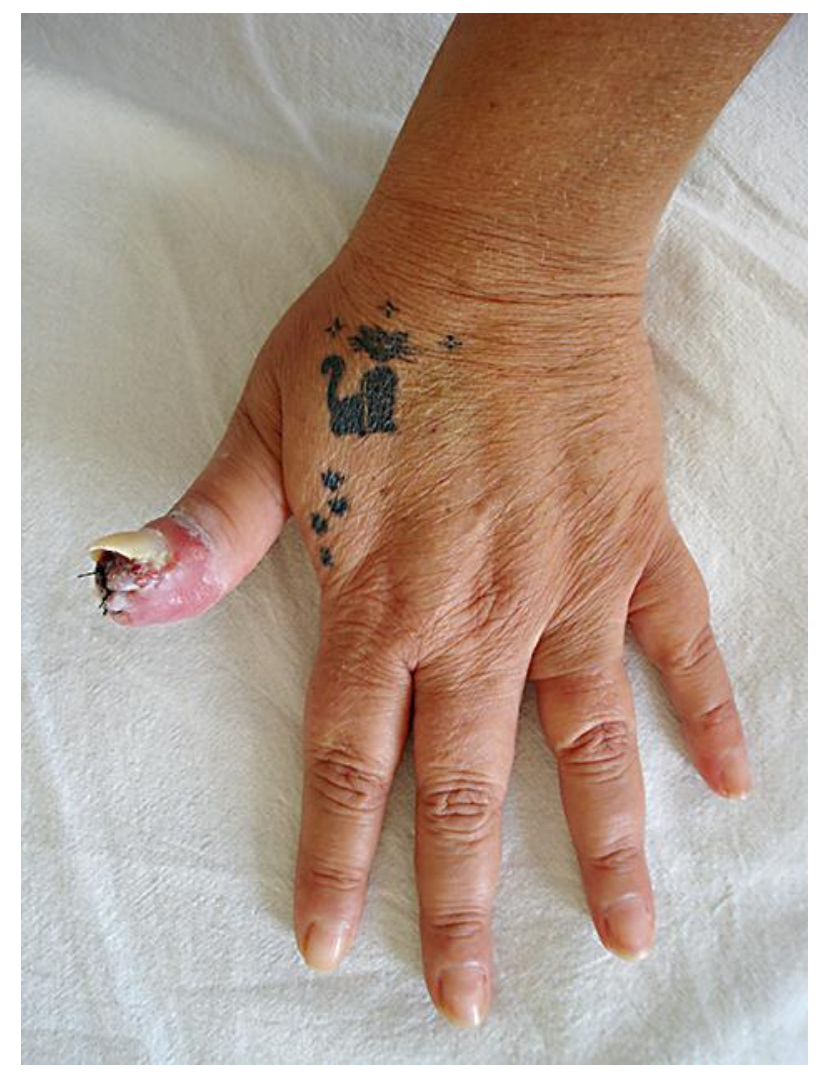

Fig. 1. Edema and significant erythema in the distal phalanx of the left thumb.

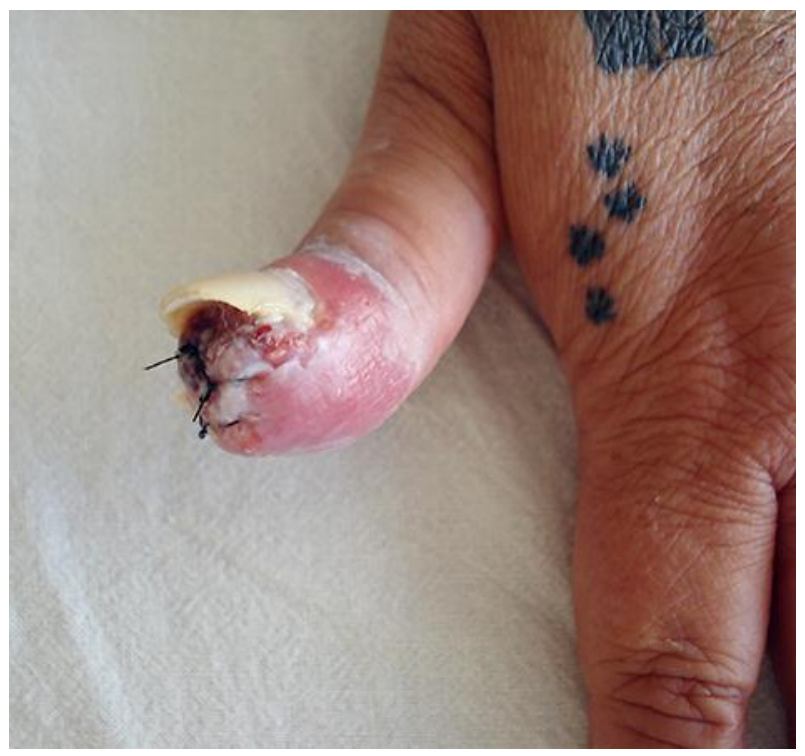

Fig. 2. Detail of the lesion with a crusted ulcerated area (where the biopsy was performed) in its distal portion, causing detachment of the nail plate after the skin biopsy. 


\section{DOI: 10.1159/000362184}

(C) 2014 S. Karger AG, Base

Aguinaga et al.: Sporotrichosis with Bone Involvement: An Alert to an Occupationa Disease

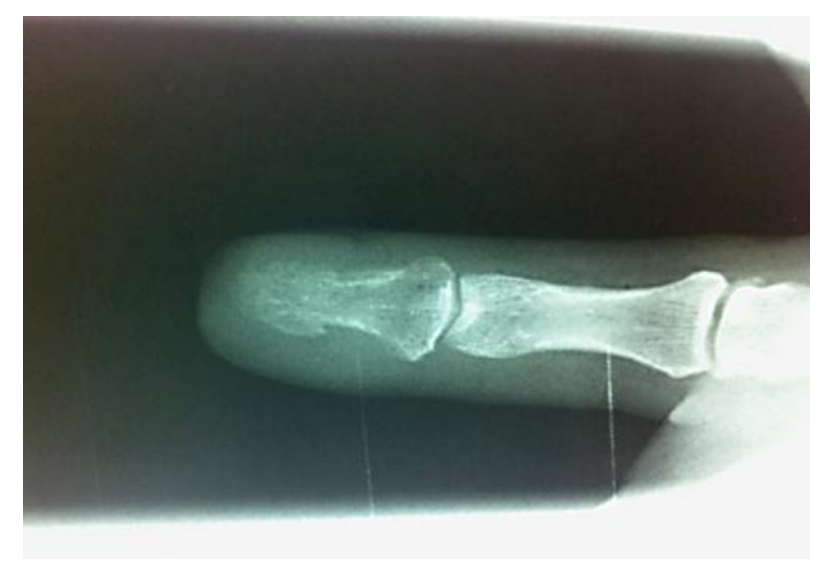

Fig. 3. Radiography showing the bone alteration (osteomyelitis) in the distal phalanx. 